\title{
Resveratrol reverses temozolomide resistance by downregulation of MGMT in T98G glioblastoma cells by the NF-кB-dependent pathway
}

\author{
HUIYONG HUANG* ${ }^{*}$ HONG LIN* ${ }^{*}$ XIANG ZHANG and JUAN LI \\ Department of Neurosurgery, Xijing Institute of Clinical Neuroscience, \\ Fourth Military Medical University, Xi'an 710032, P.R. China
}

Received November 18, 2011; Accepted January 30, 2012

DOI: $10.3892 /$ or.2012.1715

\begin{abstract}
Glioblastoma multiforme (GBM) is the most common intracranial tumor, with a dismal prognosis. Although temozolomide (TMZ)-based chemotherapy following neurosurgery has been proven to be effective, not all patients benefit clinically because of $\mathrm{TMZ}$ resistance. Given that protein expression of $\mathrm{O}(6)$-methylguanine-DNAmethyltransferase (MGMT) is the most important determinant of TMZ resistance, great efforts have been made to suppress it by regulating MGMT-related transcription factors. The study presented here demonstrates that resveratrol, a natural polyphenol, is able to reverse TMZ resistance of glioblastoma T98G cells which have relatively high MGMT activity. The data showed that combination treatment with TMZ and resveratrol resulted in an enhanced antitumor potential of TMZ, decreased the $50 \%$ inhibiting concentration (IC50) of TMZ and increased the induction of apoptosis in TMZ-resistant T98G cells. Hoechst 33258 staining revealed increased apoptotic morphology, such as chromatin aggregation and nuclear and cytoplasmic condensation, in cells receiving combination treatment. Western blot analysis manifested a significant decreased intracellular content and nuclear translocation of $\mathrm{NF}-\kappa \mathrm{B}$ and increased cleavage of caspase -3 in cells exposed to combination treatment, compared to those in cells treated with
\end{abstract}

Correspondence to: Dr Xiang Zhang, Department of Neurosurgery, Xijing Institute of Clinical Neuroscience, Fourth Military Medical University, 17 Changle Western Road, Xi'an 710032, P.R. China

E-mail: hhy791129@126.com

*Contributed equally

Abbreviations: GBM, glioblastoma multiforme; TMZ, temozolomide; MGMT, O(6)-methylguanine-DNA-methyltransferase; NF-кB, nuclear factor- $\kappa \mathrm{B}$; GSTs, glutathione-S-transferases; O(6)BG, O6-benzylguanine; STZ, streptozotocin

Key words: glioblastoma, temozolomide, MGMT, resveratrol, $\mathrm{NF}-\kappa \mathrm{B}$
$\mathrm{TMZ}$ alone. In addition, recombinant expression of $\mathrm{NF}-\kappa \mathrm{B}$ subunit p65 remarkably promoted nuclear translocation of $\mathrm{NF}-\kappa \mathrm{B}$ and abolished the TMZ-resistance reversal induced by combination treatment, suggesting an underlying NF- $\mathrm{B}$ dependent mechanism. Our study improved the knowledge on the mechanism of TMZ resistance and suggested a novel strategy for TMZ-based chemotherapy in glioblastoma patients.

\section{Introduction}

Prognosis of glioblastoma multiforme (GBM), the most common type of neoplasm in central nervous system (CNS), is dismal despite aggressive surgical resection and adjuvant chemotherapy. GBM patients often die within approximately 12 months after preliminary diagnosis $(1,2)$. Temozolomide (TMZ), an orally administered alkylating agent, has been suggested as a promising chemotherapeutic candidate. Some randomized clinical trials evidenced that TMZ-based chemotherapy could significantly improve quality of life and prolong survival of glioblastoma patients. Clinical benefit varies depending on the TMZ regimen obtained and personal genetic heterogeneity. Median survival of patients receiving TMZ concurrently with radiotherapy followed by adjuvant TMZ ranges from 14.6 to 25.5 months, compared with 12.1 months (3) in those who only received conventional radiotherapy. Two-year survival of patients receiving TMZ treatment ranges from 26.5 (4) to $46 \%(5,6)$, compared with $10.4 \%$ (4) in those who only received conventional radiotherapy. However, it is suggested that about 58\% (7) of GBM patients could not benefit from TMZ therapy, principally because of their intracellular high activity of $\mathrm{O}(6)$-methylguanine DNA-methyltransferase (MGMT).

MGMT, a DNA repair enzyme, is able to specifically remove the methyl group from O-6 positions of guanine residues which is added by TMZ. It is suggested that protein expression of MGMT is the most important determinant of TMZ-resistance (8). Prognosis of patients with high MGMT expression is much poorer than those with low or undetectable MGMT expression (9). Transcription of MGMT is modulated by its promoter methylation and a set of transcription factors. Since drugs with potential for specially methylating 
MGMT promoter do not currently exist, better understanding of MGMT-regulating proteins is very meaningful. To date, several mechanisms including NF- $\mathrm{B}$, p53, AP-1, Sp1 and estrogen receptor-mediated signaling pathways have been suggested to be involved in the transcription regulation of MGMT, however, $\mathrm{NF}-\kappa \mathrm{B}$ is the most important regulator. There are two NF- $\kappa \mathrm{B}-$ binding sites in the promoter of MGMT: one is at -766 (MGMT- $\kappa \mathrm{B}-1$ ) and the other is at -90 (MGMT$\kappa \mathrm{B}-2)$. A previous study showed that transient transfection of human embryo kidney HEK293 cells with the NF- $\mathrm{B}$ subunit p65 would induce a 55-fold increase in MGMT expression, whereas addition of the $\mathrm{NF}-\kappa \mathrm{B}$ inhibitor $\delta \mathrm{NI} \kappa \mathrm{B}$ (N-terminal domain of $\mathrm{I} \kappa \mathrm{B} \alpha$ ) wholly abrogates the augmented expression of MGMT (10). Therefore, downregulation of NF- $\mathrm{B}-\mathrm{MGMT}$ pathway is a feasible strategy to overcome TMZ-resistance.

It has been found out that a number of cytokines and chemicals could regulate the expression and activation of NF- $\kappa \mathrm{B}$. In the classical pathway, I $\kappa \mathrm{B}$ proteins conjugate with $\mathrm{NF}-\kappa \mathrm{B}$ and mask its nuclear location sequence (NLS) thus restricting its over-activation. Paclitaxel, interleukin-1 (IL-1), tumor necrosis factor- $\alpha$ (TNF- $\alpha$ ), growth factors including epidermal growth factor (EGF) and transforming growth factor- $\alpha$ (TGF- $\alpha$ ) induce the phosphorylation and degradation of $\mathrm{I} \kappa \mathrm{B} \alpha$ proteins through phosphatidylinositol-3-kinase (PI3K)-Akt pathway, which set $\mathrm{NF}-\kappa \mathrm{B}$ free and facilitates its subsequent nuclear translocation. In a preliminary study $(11,12)$, we found that Notch-1 activation-dependent p53 restoration contributes to resveratrol-induced apoptosis in glioblastoma A172 and T98G cells, and the activation of p53 strongly suppresses the expression of MGMT, suggesting that it could be employed in the TMZ-resistance reversal via their regulation on $\mathrm{NF}-\kappa \mathrm{B}$ MGMT signaling. However, whether resveratrol plays a role on the phosphorylation and degradation of $\mathrm{I} \kappa \mathrm{B}$ proteins is unclear. Given that $\mathrm{NF}-\kappa \mathrm{B}$ is an important positive transcriptional regulator of MGMT while p53 is the most important negative transcriptional regulator of MGMT, we investigated whether resveratrol can decrease MGMT expression and subsequently reverse temozolomide-resistance through repressed $\mathrm{NF}-\kappa \mathrm{B}$ expression and increased p53 expression in the following experiments.

To address this hypothesis, the current study was performed in the typical glioblastoma cell line T98G, which abundantly expresses MGMT and is resistant to TMZ-based chemotherapy. Inhibiting concentration (50\%) (IC50) of TMZ and the extent of apoptosis-induction were assessed to affirm whether the combination treatment of resveratrol and TMZ was able to reverse TMZ-resistance. Evaluation of intracellular expression and subcellular distribution of NF- $\kappa \mathrm{B}$ and MGMT was carried out to investigate the underlying mechanism of possible TMZ-resistance reversal.

\section{Materials and methods}

Agents. Temozolomide (Santa Cruz Biotechnology, Santa Cruz, CA) was dissolved in dimethyl sulphoxide (DMSO) (Gibco/ Invitrogen, Grand Island, NY, USA) to produce a $100 \mathrm{mM}$ stock solution. Resveratrol (Santa Cruz Biotechnology) was dissolved in dimethyl sulfoxide (DMSO) (Gibco/Invitrogen) to produce a 100-mM stock solution. Before each experiment, temozolomide and resveratrol were dissolved in fresh
Dulbecco's modified Eagle's medium (DMEM) (Sigma Chemical Co., St. Louis, MO, USA) to obtain 100 and $100 \mu \mathrm{M}$ working solutions, respectively. For temozolomide solutions, the final DMSO concentration did not exceed $1 \%(\mathrm{v} / \mathrm{v})$, so cell growth was not affected.

Cell lines and cell culture. Human glioblastoma cell strains T98G, A172, U87MG and U251MG cells (purchased from Cell Bank of Fourth Military Medical University, China) were cultured in DMEM medium supplemented with $10 \%$ fetal bovine serum (FBS) (BioSun Sci\&Tech Co., Ltd., Shanghai, China) in a $37^{\circ} \mathrm{C}$ incubator with a humidified atmosphere of $5 \% \mathrm{CO}_{2}-95 \% \mathrm{O}_{2}$. Twenty-four hours before the experiments, cells were transferred to serum free medium. Temozolomide and resveratrol were added to the culture medium to reach the final concentrations as indicated in the following experiments.

MTT assay. Cell viabilities were determined by methylthiazolyl-tetrazolium (MTT) assay. Briefly, T98G cells were cultured in different 96-well plates (Greiner Bio-One $\mathrm{GmbH}$, Frickenhausen, Germany) at a density of $5 \times 10^{3}$ cells/well. In treatment groups, temozolomide was added to culture medium at final concentrations of $50,100,200$ or $400 \mu \mathrm{M}$, resveratrol was added at a final concentration of 100, 200, 400 or $800 \mu \mathrm{M}$. In the combination treatment group, resveratrol $(100 \mu \mathrm{M})$ was added to the culture medium in combination with temozolomide $(100 \mu \mathrm{M})$. After incubation for 24 and $72 \mathrm{~h}$, MTT (Sigma) solved in PBS was added to each well at a final concentration of $5 \mathrm{mg} / \mathrm{ml}$ and then incubated at $37^{\circ} \mathrm{C}$ for $4 \mathrm{~h}$. The water-insoluble dark blue formazan crystals that formed during MTT cleavage in actively metabolizing cells were dissolved in DMSO. The optical density was measured at a wavelength of $490 \mathrm{~nm}$ with a Bio-Rad 680 microplate reader (Bio-Rad Laboratories, Hercules, CA, USA). The reduction in viability of the $\mathrm{T} 98 \mathrm{G}$ cells in different treatment groups was expressed as the percentage compared with temozolomide and resveratrol-free control cells. All experiments were done in triplicate.

Hoechst 33258 nuclear staining. Briefly, cells $\left(1 \times 10^{5}\right)$ were plated in 6-well plates, and treated with temozolomide and/ or resveratrol for $72 \mathrm{~h}$, respectively. Cells were then washed in PBS (0.01 M, pH 7.4) and fixed in $70 \%$ ethanol for $2 \mathrm{~h}$ at $4^{\circ} \mathrm{C}$. Cell nuclei were stained with Hoechst $33258(5 \mu \mathrm{g} / \mathrm{ml}$; Sigma). After final washing in PBS, the changes in nuclear morphology were visualized by fluorescence microscopy (Leica Microsystems, Wetzlar, Germany) using excitation at wavelength of 330 and $380 \mathrm{~nm}$.

Annexin V/propidium iodide. To determine the extent of apoptosis-induction, Annexin $\mathrm{V}$ assays were performed using an apoptosis detection kit (Annexin V-FITC/PI Staining Kit; Immunotech Co., Marseille, France). Briefly, cells $\left(1 \times 10^{5}\right)$ were plated in 6-well plates, and treated with temozolomide and/or resveratrol for $72 \mathrm{~h}$, respectively. Cells were harvested by scraping, washed in cold PBS (0.01 M, pH 7.4), incubated for 15 min with fluorescein-conjugated Annexin V and propidium iodide, and analyzed using a FACScan flow cytometer equipped with the FACStation data management system 
running CellQuest software (Becton-Dickinson, San Jose, CA, USA). Annexin V-positive cells were considered to be apoptotic, while cells that were both PI and Annexin V-negative were considered normal.

Western blot analysis. Protein extracts were prepared by lysing the cells in RIPA buffer $(150 \mathrm{mM} \mathrm{NaCl}, 1 \%$ Non-idet $\mathrm{P}-40,0.5 \%$ sodium deoxycholate, $0.1 \% \mathrm{SDS}, 50 \mathrm{mM}$ Tris- $\mathrm{HCl}$, pH 8.0) containing $10 \mathrm{mM}$ ethylenediamine tetraacetic acid (EDTA), $1 \mathrm{mM}$ sodium orthovanadate and $1 \mathrm{mM}$ phenylmethylsulfonylfluoride (PMSF; all from Sigma) for $30 \mathrm{~min}$ at $4^{\circ} \mathrm{C}$. Samples were then centrifuged at $14,000 \mathrm{x}$ g for $25 \mathrm{~min}$ at $4^{\circ} \mathrm{C}$. The protein concentration in the supernatant was determined by BCA Protein Assay Kit (HyClone-Pierce, Logan, UT, USA). Equivalent amounts (10 $\mu \mathrm{g}$ protein/lane) of protein lysates were separated by $12 \%$ SDS-polyacrylamide gel electrophoresis and transferred onto a nitrocellulose membrane $(0.22 \mu \mathrm{m}$, Invitrogen) in a transfer tank (Bio-Rad Laboratories) using the submerged method. The membrane was blocked for $2 \mathrm{~h}$ at room temperature in PBS containing 0.1\% Tween-20 (Sigma) and 5\% non-fat dried milk (Carnation City, OH, USA), and then incubated with the primary antibody in dilution buffer (19 TBS, $0.1 \%$ Tween-20 with 5\% BSA) with gentle agitation overnight at $4{ }^{\circ} \mathrm{C}$. Primary antibodies were: anti-MGMT (diluted 1:1000, mouse monoclonal), anti-NF- $\mathrm{KB}$ (diluted 1:1000, mouse monoclonal), anti-IкB $\alpha$ (diluted 1:1000, mouse monoclonal), anti-phospho-IкB $\alpha$ (39A1431) (diluted 1:1000, mouse monoclonal), anti-cleaved caspase-3 p11 (h176) (diluted $1: 1000$, goat polyclonal), and anti- $\beta$-actin (diluted $1: 1000$, mouse monoclonal). Then, the membranes were washed in dilution buffer (1X TBS, $0.1 \%$ Tween-20 with 5\% BSA) and incubated for $1 \mathrm{~h}$ with horseradish-peroxidase (HRP)conjugated secondary antibodies, and finally developed by an ECL system (Cell Signaling Technology, Beverly, MA, USA). Secondary antibodies were: HRP-conjugated anti-goat IgG (diluted 1:2000) and HRP-conjugated anti-mouse IgG (diluted 1:2000). All the antibodies were purchased from Santa Cruz Biotechnology. The western blotting was performed following the Laemmli method and the grayscale values of each band on the blots were measured using BandScan4.3.

Statistical analysis. Data are expressed as the mean \pm the standard error of mean (SEM) of separate experiments. All data were tested for significance by one-way analysis of variance (ANOVA) followed by Fisher's post hoc test using SPSS13.0 software. Results with a P-value $<0.05$ were considered statistically significant.

\section{Results}

Expression of MGMT protein in T98G, U87MG, A172 and SHG44 cells. The expression of MGMT protein was high in T98G cells, and very low or hardly detected in U87MG, A172 and SHG44 cells (Fig. 1). T98G cells were chosen to carry out the following experiments.

Resveratrol strengthens the TMZ suppression of cell viability in glioblastoma T98G cells. MTT assay suggested that T98G glioblastoma cells are insensitive to TMZ treatment. When exposed to TMZ $(100 \mu \mathrm{M})$ for 24 and $72 \mathrm{~h}$, the cell viability

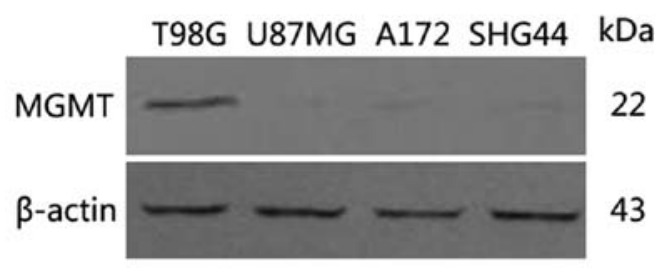

Figure 1. MGMT expression in glioblastoma cell lines. MGMT is positively expressed in T98G cells while negatively or weakly expressed in U87MG, A172 and SHG44 cells.
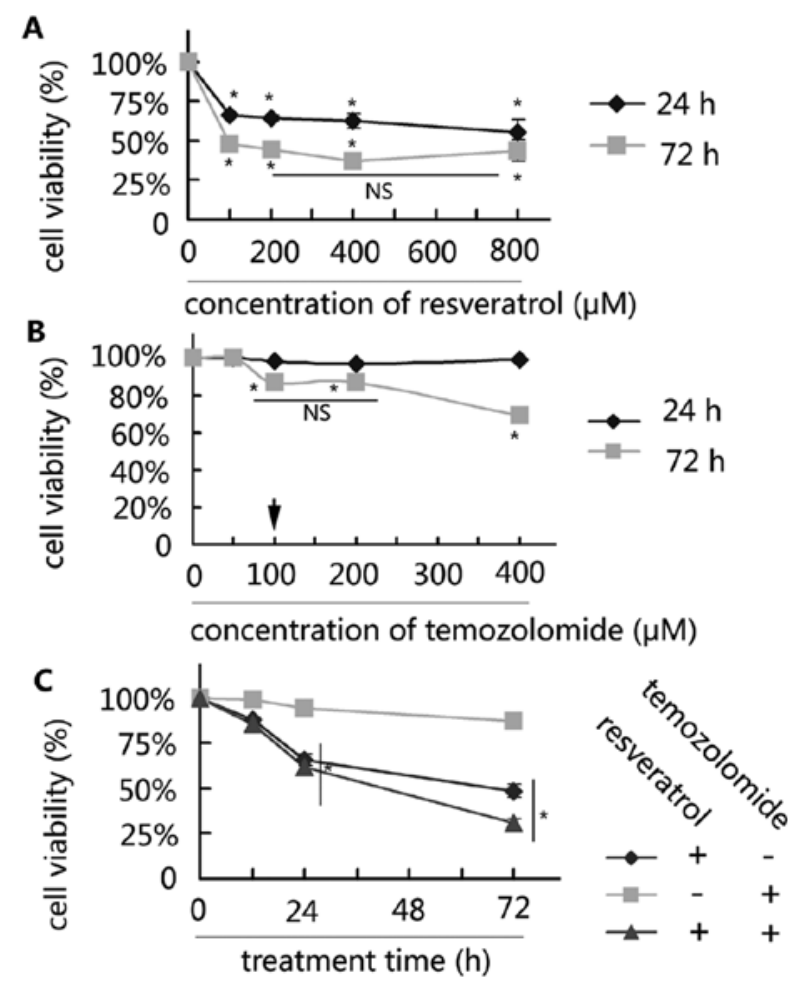

Figure 2. Growth-inhibitory effects of resveratrol and temozolomide on the cell viability of glioblastoma T98G cells. (A) Resveratrol remarkablly inhibited cell viability of glioblastoma T98G cells in a concentration-dependent manner. (B) Temozolomide remarkably inhibited the cell viability of glioblastoma T98G cells in a concentration-dependent manner. (C) Simultaneous treatment with resveratrol enhanced temozolomide-induced growth inhibition on glioblastoma T98G cells. Bars, SEM; ${ }^{\mathrm{P}}<0.05$ vs. vehicle control; NS, non-significant; red arrow, clinically relavant concentration.

of $\mathrm{T} 98 \mathrm{G}$ cells slowly decreased to $98.11 \pm 1.1 \%$ and $86.9 \pm 2.1 \%$ compared to the vehicle-treatment controls, respectively (Fig. 2A); while exposed to resveratrol $(100 \mu \mathrm{M})$ for 24 and $72 \mathrm{~h}$, the viability of T98G cells was significantly decreased to $65.8 \pm 0.6 \%$ and $48.4 \pm 2.2 \%$, respectively (Fig. $2 B$ ). By contrast, the TMZ and resveratrol combination treatment $(100 \mu \mathrm{M}$ $\mathrm{TMZ}+100 \mu \mathrm{M}$ resveratrol for 12,24 and $72 \mathrm{~h}$ ) significantly decreased the cell viabilities of T98G to $85.5 \pm 2.8 \%, 61.4 \pm 4.5 \%$ and $30.8 \pm 2.8 \%$, respectively (Fig. $2 \mathrm{C}$ ), which were statistically different from those treated with TMZ $(100 \mu \mathrm{M})$ alone for 24 and $72 \mathrm{~h}(\mathrm{P}=0.000$, analyzed by unpaired Student's t-test). The 50\% inhibiting concentrations (IC50) of TMZ applied alone or in combination with resveratrol at 24 and $72 \mathrm{~h}$ were $2.5 \mathrm{M}, 3.2 \mathrm{nM}, 4.1 \mathrm{nM}$ and $33.7 \mu \mathrm{M}$. Decreased IC50 of TMZ 


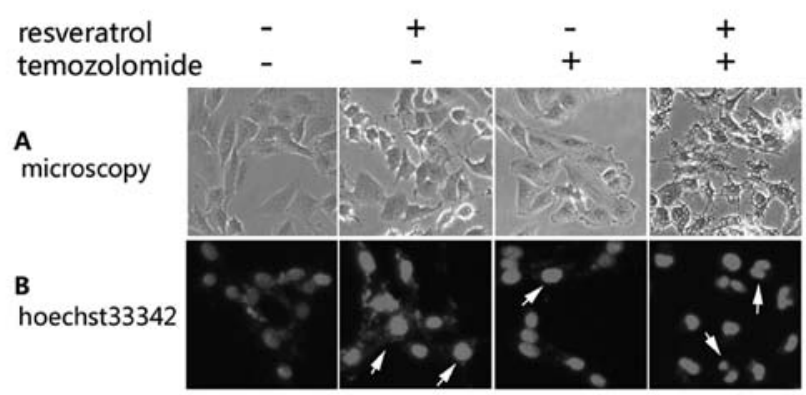

Figure 3. Effects of resveratrol and temozolomide on the cellular and nuclear morphology of glioblastoma cells. (A) Resveratrol $(100 \mu \mathrm{M}, 72 \mathrm{~h})$ and temozolomide ( $100 \mu \mathrm{M}, 72 \mathrm{~h})$ mono-treatments slightly induced contraction and condensation of glioblastoma T98G cells, respectively. Combination treatment with resveratrol $(100 \mu \mathrm{M}, 72 \mathrm{~h})$ and temozolomide $(100 \mu \mathrm{M}$, $72 \mathrm{~h}$ ) induced conspicuous apoptotic cellular and nuclear morphology. (B) Reaveratrol $(100 \mu \mathrm{M}, 72 \mathrm{~h})$ and temozolomide $(100 \mu \mathrm{M}, 72 \mathrm{~h})$ mono-treatments led to condensation of apoptotic nuclues and increased their diopter in glioblastoma cells, respectively. Combination treatment with resevratrol $(100 \mu \mathrm{M}, 72 \mathrm{~h})$ and temozolomide $(100 \mu \mathrm{M}, 72 \mathrm{~h})$ resulted in bud-formation of apoptotic nucleus.

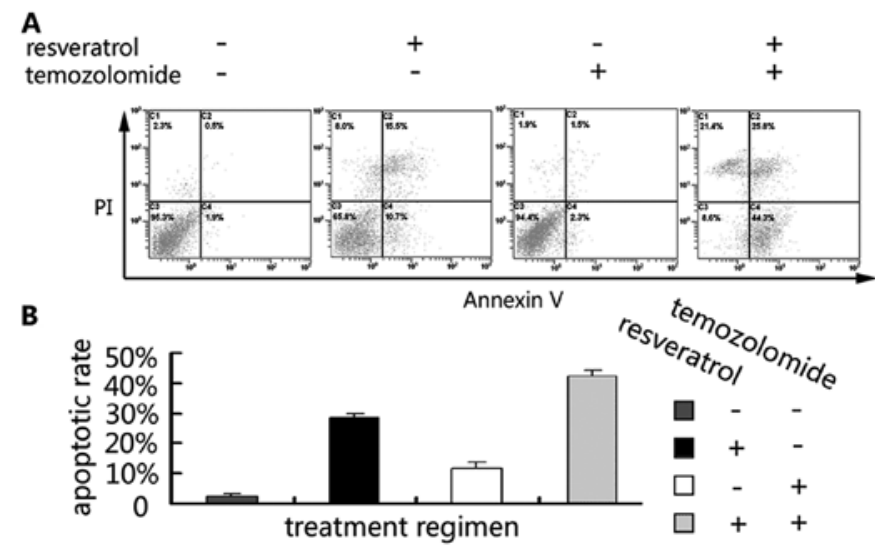

Figure 4. Annexin V-FITC/PI-based apoptosis-detection by flow cytometry. (A) Resveratrol $(100 \mu \mathrm{M}, 24 \mathrm{~h})$ and temozolomide (100 $\mu \mathrm{M}, 24 \mathrm{~h})$ monotreatments induced pronounced apoptosis in glioblastoma T98G cells, respectively. Combination treatment with resveratrol $(100 \mu \mathrm{M}, 24 \mathrm{~h})$ and temozolomide (100 $\mu \mathrm{M}, 24 \mathrm{~h}$ ) induced remarkable apoptosis. (B) Statistical analysis.

suggested that additionally applied resveratrol strengthened the toxicity of TMZ.

Resveratrol increases TMZ-induced apoptosis of glioblastoma T98G cells. Treatment with TMZ $(100 \mu \mathrm{M})$ or combination treatment with resveratrol $(100 \mu \mathrm{M})$ and TMZ $(100 \mu \mathrm{M})$ for $72 \mathrm{~h}$ is shown in Fig. 3. In TMZ monotherapy group, T98G cell slightly experienced apoptotic nuclear features such as aggregation and marginality of chromatin and nuclear and cytoplasmic condensation. In combination treatment group, the morphologic changes were much more severe than the monotherapy group, a number of nuclei displayed a lobulated appearance, many were fractured and scattered out of the cells, even the bud-formation of the apoptotic nucleus.

In order to verify the toxicity of TMZ on glioblastoma cells, we performed Annexin V assays and compared the ability of TMZ monotherapy and its combination treat-

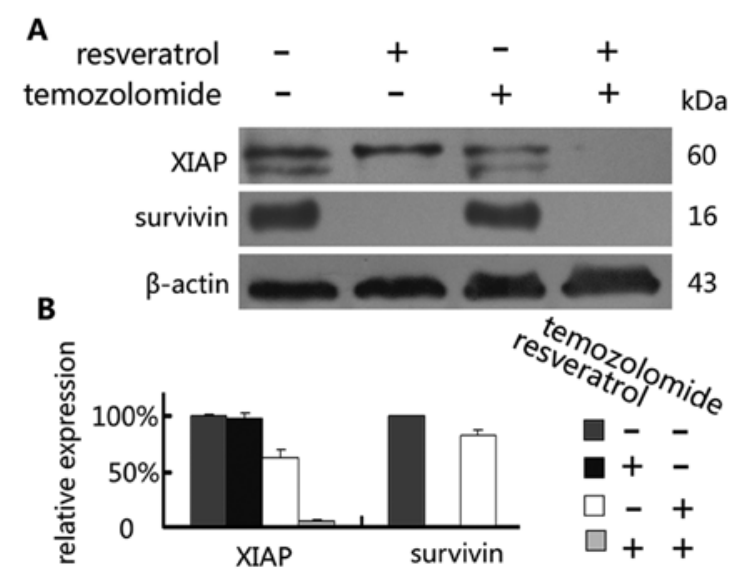

Figure 5. Effects of resveratrol and temozolomide on the expression of inhibitor of apoptosis family proteins XIAP and survivin in glioblastoma T98G cells. (A) Resveratrol (100 $\mu \mathrm{M}, 24 \mathrm{~h})$ and temozolomide (100 $\mu \mathrm{M}, 24 \mathrm{~h})$ mono-treatments repressed expression of XIAP and survivin in glioblastoma T98G cells, respectively. Combination treatment with resveratrol $(100 \mu \mathrm{M}$, $24 \mathrm{~h}$ ) and temozolomide (100 $\mu \mathrm{M}, 24 \mathrm{~h})$ completely blocked their expression. (B) Statistical analysis.

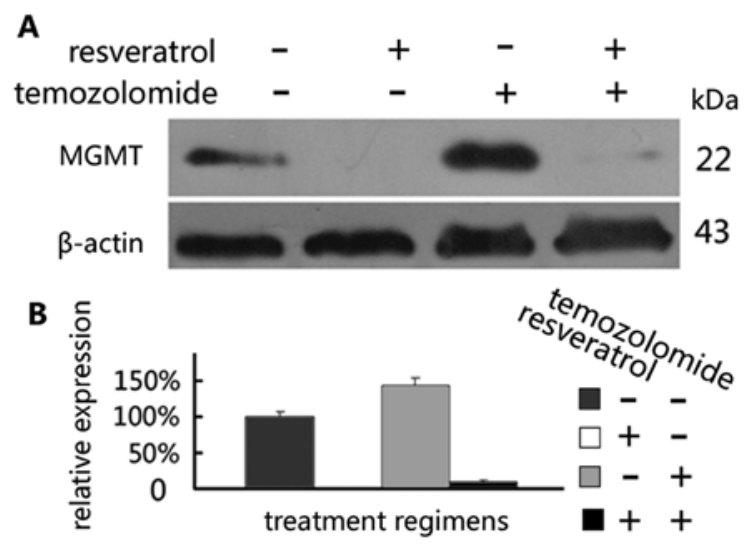

Figure 6. Effects of resveratrol and temozolomide on the expression of MGMT protein in glioblastoma T98G cells. (A) Resveratrol (100 $\mu \mathrm{M}, 24 \mathrm{~h})$ completely blocked while temozolomide $(100 \mu \mathrm{M}, 24 \mathrm{~h})$ notably upregulated MGMT expression in glioblastoma T98G cells, respectively. Combination treatment with resveratrol $(100 \mu \mathrm{M}, 24 \mathrm{~h})$ and temozolomide $(100 \mu \mathrm{M}, 24 \mathrm{~h})$ significantly repressed temozolomide-induced upregulation of MGMT expression. (B) Statistical analysis.

ment with resveratrol. After exposure to TMZ $(100 \mu \mathrm{M})$ or combination treatments with TMZ $(100 \mu \mathrm{M})$ and resveratrol $(100 \mu \mathrm{M})$ for $24 \mathrm{~h}$, significant apoptosis was induced in T98G cells (Fig. 4A). The apoptotic rates in combination treatment groups were higher than TMZ monotherapy $(42.5 \pm 1.8 \mathrm{vs}$. $11.5 \pm 2.3 \%$ ) (Fig. 4B). This suggests resveratrol significantly attenuates TMZ-induced apoptosis.

Resveratrol strengthens TMZ suppression of the expression of inhibitor of apoptosis family proteins XIAP and survivin. To further explore the molecular mechanisms underlying the effect of resveratrol on TMZ-resistance glioblastoma T98G cells, we quantified changes in the expression levels of inhibitor of apoptosis family proteins during treatment. After treatment with resveratrol $(100 \mu \mathrm{M})$ and TMZ $(100 \mu \mathrm{M})$ for 


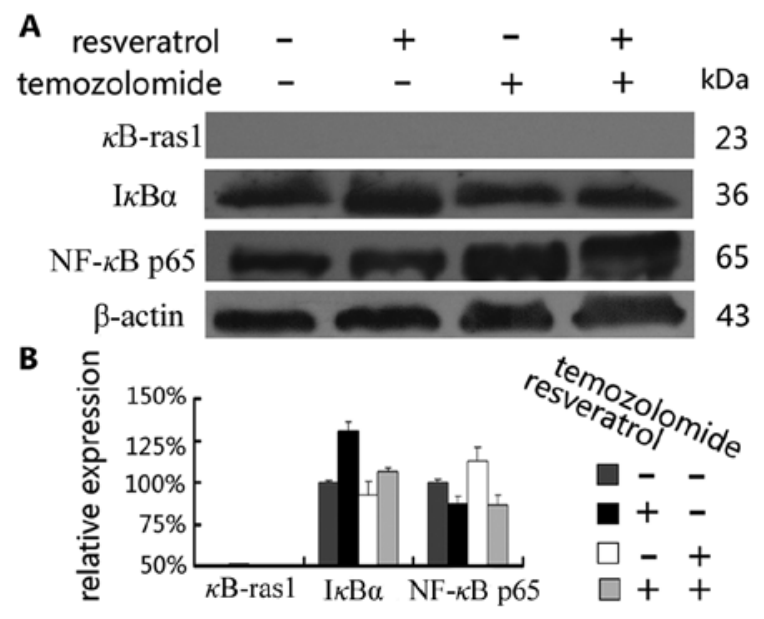

Figure 7. Effects of resveratrol and temozolomide on the expression of proteins in NF- $\mathrm{B}$ signaling pathway in glioblastoma T98G cells. (A) Neither resveratrol $(100 \mu \mathrm{M}, 24 \mathrm{~h})$ nor temozolomide $(100 \mu \mathrm{M}, 24 \mathrm{~h})$ treatment alone or in comination, rarely affected the expression of $\kappa \mathrm{B}$-ras1. Resveratrol increased $\mathrm{I} \kappa \mathrm{B} \alpha$ expression and decreased NF- $\kappa \mathrm{B}$ expression while temozolomide effect was the opposite. Combination treatment with resveratrol and temozolomide significantly repressed temozolomide-induced upregulation of NF- $\kappa \mathrm{B}$ expression. (B) Statistical analysis.

$24 \mathrm{~h}$, respectively, the expression of XIAP and survivin protein levels were reduced in the malignant glioma T98G cells (Fig. 5A). Resveratrol suppressed the expression of survivin more than TMZ, while TMZ inhibited the ability of XIAP expression better than resveratrol. Exposure to resveratrol $(100 \mu \mathrm{M})$ for $24 \mathrm{~h}$, XIAP expression was not significantly reduced in glioblastoma $\mathrm{T} 98 \mathrm{G}$ cells $(\mathrm{P}=0.62)$, and survivin protein expression was almost completely blocked $(\mathrm{P}=0.001)$ (Fig. 5B). Exposure to TMZ $(100 \mu \mathrm{M})$ for $24 \mathrm{~h}$, survivin expression was not significantly lower in glioblastoma T98G cell $(\mathrm{P}=0.49)$, and XIAP protein expression was significantly reduced to $62.3 \pm 4.8 \%(\mathrm{P}=0.001)$ (Fig. $5 \mathrm{~B})$. By contrast, exposure to resveratrol $(100 \mu \mathrm{M})$ and TMZ $(100 \mu \mathrm{M})$ combined for $24 \mathrm{~h}$, XIAP $(\mathrm{P}=0.001)$ and survivin protein $(\mathrm{P}=0.001)$ had a very significant reduction. Therefore, the combination treatment resveratrol and TMZ-induced apoptosis, in glioblastoma T98G cells is likely to be blocked by the co-inhibitor of apoptosis protein family.

Resveratrol strengthens the TMZ suppression of MGMT protein. Western blot analysis showed that (Fig. 6), exposure to $\mathrm{TMZ}(100 \mu \mathrm{M})$ for $24 \mathrm{~h}$ significantly increased the protein expression of MGMT (149.6 $\pm 8.3 \%, \mathrm{P}=0.001)$, However, its combination treatment with resveratrol $(100 \mu \mathrm{M})$ strikingly downregulated the protein expression of MGMT $(\mathrm{P}=0.001)$. Experimental results showed that resveratrol could completely block not only endogenous protein expression of MGMT but also increasing protein expression of MGMT by the TMZ-induced in glioblastoma T98G cells. This important molecular mechanism to reverse TMZ-resistance with resveratrol blocked the protein expression of MGMT in glioblastoma T98G cells.

Involvement of the NF- $\mathrm{B}-M G M T$ pathway in the therapeutic potential of combination with resveratrol and TMZ treatment.
The underlying mechanism was investigated by detecting expression levels of proteins engaged in the NF- $\mathrm{B}-\mathrm{MGMT}$ pathway (Fig. 7). Exposure to TMZ (100 $\mu \mathrm{M})$ for $24 \mathrm{~h}$ hardly affected the expression of $\kappa \mathrm{B}$-ras1 and $\mathrm{I} \kappa \mathrm{B} \alpha(98.3 \pm 4.6 \%$, $\mathrm{P}=0.57)$, but significantly increased the expression of $\mathrm{NF}-\kappa \mathrm{B}$ subunit p65 $(109.2 \pm 2.8 \%, \mathrm{P}=0.03)$. By contrast, exposure to combination with TMZ $(100 \mu \mathrm{M})$ and resveratrol $(100 \mu \mathrm{M})$ treatment hardly affected the expression of $\kappa \mathrm{B}$-ras1 and $\mathrm{I} \kappa \mathrm{B} \alpha$ (105.5 $\pm 7.6 \%, \mathrm{P}=0.07)$, but significantly reduced the expression of $\mathrm{NF}-\kappa \mathrm{B}$ subunit p65 $(75.5 \pm 2.3 \%, \mathrm{P}=0.001)$. This suggests resveratrol can negative regulate $\mathrm{NF}-\kappa \mathrm{B}$ by upregulating the express of $\mathrm{I} \kappa \mathrm{B} \alpha$, but this may be only the process in inducing apoptosis of malignant glioma, rather than reducing expression of MGMT by downregulated NF-кB.

\section{Discussion}

Resistance to TMZ-based chemotherapy is complex and not yet clarified. Some mechanisms have been revealed, principally the failure of effective drug uptake to achieve a sufficient concentration for enough exposure time within tumor parenchyma and the rapid repair of DNA damage which are induced by TMZ. The former could be overcome by regional administration through an Ommaya reservoir which allows TMZ at an adequate concentration being infused directly toward tumor cavity. The latter may be related to two major pathways, one is glutathione-S-transferases (GSTs)mediated pathway and the other is $\mathrm{O}(6)$-methylguanine DNA-methyltransferase (MGMT)-mediated pathway. Given that both pathways prevalently exist in GBMs (13), TMZ monochemotherapy may not respond completely in all the GBMs and thus different combinations of TMZ with other drugs are encouraged to diminish the unexpected DNA repair. It is suggested that inhibitor of GSTs, such as isocyanates and agaricus bisporus lectin, may deplete bioactive glutathione or suppress its nuclear transfer so as to block GSTs-mediated DNA repair (14). However, these inhibitors usually lead to unfavorable systematic cytotoxicity which restricts their clinical implication. In contrast, drugs targeting the inhibition of MGMT activity are currently actively studied. Many MGMT inhibitors have been developed and introduced into the experimental and clinical studies to produce an increase of sensitivity to TMZ in the treatments for glioblastoma or other tumors. O6-benzylguanine $(\mathrm{O}(6) \mathrm{BG})(15,16)$, streptozotocin (STZ) (17), and O6-(4-bromothenyl) guanine (PaTrin-2) (18) are three most popular agents applied in combination with TMZ. All of them are able to deplete intracellular MGMT content for variable duration in a pseudosubstrate inhibition manner. However, although they can increase cancer cell lesion burden, serious genotoxicity effect and systematic toxicity have been reported. Therefore, further studies on drugs which have been demonstrated to be well tolerated and with low systematic cytotoxic by long-term clinical practice have potential in modulating MGMT and its regulating proteins, are warranted to see whether they can decrease MGMT activity and sensitize glioblastoma cells to TMZ chemotherapy with less side effects.

Given that nuclear translocation of NF- $\mathrm{BB}$ subunit p65 is the key event of its activation and resveratrol is an effective 
inhibitor of this subcellular redistribution, the competence of resveratrol to suppress NF- $\mathrm{B}-\mathrm{MGMT}$ pathway and to overcome TMZ-resistance is of clinical significance. Current study validated the synergistic toxicity of TMZ and resveratrol combination treatment on the originally TMZ-resistant glioblastoma T98G cells. This combination strategy potentiated TMZ to markedly inhibit the proliferation of glioblastoma cells with lower IC50 and to induce more apoptosis than TMZ monotherapy, as evidenced by the evaluations of phosphatidylserine externalization and caspase- 3 cleavage. In addition, the critical role of the resveratrol-suppressed NF- $\kappa$ B-MGMT pathway was demonstrated by recombinant expression of $\mathrm{NF}-\kappa \mathrm{B}$ in glioblastoma cells in which the synergistic effects were principally blocked. Our results also suggested that the expression and phosphorylation of $\mathrm{I} \kappa \mathrm{B} \alpha$ were not engaged in the suppression of NF-кB-MGMT pathway. Moreover, data suggested that this synergistic effect is more significant in T98G cells.

TMZ induces apoptosis and autophagia of GBM cells. Our results show significant apoptosis in the TMZ-treated glioblastoma cells. However, it is notable that the apoptotic rate revealed by Annexin $\mathrm{V}$ assay is far below the inhibitory rate calculated by MTT assays. A probable explanation is apoptosis-induction, which is just one of the major mechanisms for TMZ and resveratrol combination treatment-induced cell killing, and other forms of cell death including autophagia may be initiated concurrently.

Persistent exposure to TMZ is suggested to bring about acquired drug-resistance of glioblastoma cells (19). TMZ results in serious DNA damage in glioblastoma cells. When it was sensed by these cells, $\mathrm{NF}-\kappa \mathrm{B}$ pathway was activated to antagonize the endogenous pro-death signals (20). In addition, NF- $\kappa \mathrm{B}$ may also induce re-expression of MGMT and thus results in acquired TMZ-resistance of glioblastoma cells. This concept could be supported by the fact that the expression of MGMT increased in $83.3 \%$ patients who have methylated promoter and negative MGMT expression before TMZ-treatment (21). However, whether activated NF- $\kappa \mathrm{B}$ strengthens MGMT expression by directly de-methylating its promoter is not evidenced although a previous study indicates that under the chemotherapeutic stress, MGMT promoter experiences de-methylation in 27.8-61.5\% of glioblastoma

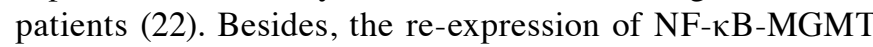
pathway, there is another explanation for acquired drug-resistance of glioblastoma cells. TMZ-based chemotherapy kills most of the MGMT-insufficient GBM cells and the residual MGMT-proficient GBM cells become the dominant population. Cancer stem cells of glioblastoma are not uniformly sensitive to TMZ (23). Some of them express high level of MGMT and have promoter methylation. This subpopulation of cancer stem cells may escape from TMZ treatment because they exist in the inner core of the tumor mass (24). In the interval of TMZ-treatment, they eventually proliferate to be the majority of glioblastoma substance and contribute to the acquired TMZ-resistance. However, no matter which mechanism works or even the the combination, the core event occurred in the acquired TMZ-resistance is the re-expression of MGMT. Therefore, resveratrol and TMZ combination treatment may offer clinical benefits for both patients with intrinsic and acquired TMZ-resistance.
In conclusion, our study showed that resveratrol and TMZ combination treatment can reverse TMZ-resistance of glioblastoma cells. This bioactivity is carried out through inhibiting the NF- $\mathrm{B}-\mathrm{MGMT}$ pathway. The blockage of nuclear translocation of $N F-\kappa B$ rather than inactivation of its classic inhibitor $\mathrm{I} \kappa \mathrm{B}$ is the critical mechanism. Data suggested a novel combination strategy for TMZ-based chemotherapy, which should be further investigated in vivo and in pre-clinical studies.

\section{Acknowledgements}

The authors would like to thank Mr. Wei Xiong for his excellent technical assistance.

\section{References}

1. DeAngelis LM: Brain tumors. N Engl J Med 344: 114-123, 2001.

2. Furnari FB, Fenton T, Bachoo RM, et al: Malignant astrocytic glioma: genetics, biology and paths to treatment. Genes Dev 21: 2683-2710, 2007.

3. Sher DJ, Henson JW, Avutu B, et al: The added value of concurrently administered temozolomide versus adjuvant temozolomide alone in newly diagnosed glioblastoma. J Neurooncol 88: 43-50, 2008.

4. Hegi ME, Diserens AC, Gorlia T, et al: MGMT gene silencing and benefit from temozolomide in glioblastoma. N Engl J Med 352: 997-1003, 2005.

5. Stupp R, Hegi ME, Neyns B, et al: Phase I/IIa study of cilengitide and temozolomide with concomitant radiotherapy followed by cilengitide and temozolomide maintenance therapy in patients with newly diagnosed glioblastoma. J Clin Oncol 28: 2712-2718, 2010.

6. Stupp R, Mason WP, van den Bent MJ, et al: Radiotherapy plus concomitant and adjuvant temozolomide for glioblastoma. $\mathrm{N}$ Engl J Med 352: 987-996, 2005.

7. Gilbert MR, Friedman HS, Kuttesch JF, et al: A phase II study of temozolomide in patients with newly diagnosed supratentorial malignant glioma before radiation therapy. Neuro Oncol 4: 261-267, 2002.

8. Yoshino A, Ogino A, Yachi K, et al: Gene expression profiling predicts response to temozolomide in malignant gliomas. Int $\mathbf{J}$ Oncol 36: 1367-1377, 2010.

9. Spiegl-Kreinecker S,Pirker C,Filipits M, et al: O6-Methylguanine DNA methyltransferase protein expression in tumor cells predicts outcome of temozolomide therapy in glioblastoma patients. Neuro Oncol 12: 28-36, 2010.

10. Lavon I, Fuchs D, Zrihan D, et al: Novel mechanism whereby nuclear factor kappaB mediates DNA damage repair through regulation of $\mathrm{O}(6)$-methylguanine-DNA-methyltransferase. Cancer Res 67: 8952-8959, 2007.

11. Lin H, Xiong W, Huang H, et al: Notch-1 activation-dependent p53 restoration contributes to resveratrol-induced apoptosis in glioblastoma cells. Oncol Rep 26: 925-930, 2011.

12. Lin H, Wang Y, Zhang X, et al: Prognostic significance of kappaB-Ras1 expression in gliomas. Med Oncol: Feb 8, 2011 (Epub ahead of print).

13. Ali-Osman F, Brunner JM, Kutluk TM, et al: Prognostic significance of glutathione S-transferase pi expression and subcellular localization in human gliomas. Clin Cancer Res 3: 2253-2261, 1997.

14. Goto S, Kamada K, Soh Y, Ihara Y, et al: Significance of nuclear glutathione S-transferase pi in resistance to anti-cancer drugs. Jpn J Cancer Res 93: 1047-1056, 2002.

15. Kokkinakis DM, Ahmed MM, Chendil D, et al: Sensitization of pancreatic tumor xenografts to carmustine and temozolomide by inactivation of their O6-methylguanine-DNA methyltransferase with O6-benzylguanine or O6-benzyl-2'-deoxyguanosine. Clin Cancer Res 9: 3801-3807, 2003.

16. Quinn JA, Jiang SX, Reardon DA, et al: Phase II trial of temozolomide plus O6-benzylguanine in adults with recurrent, temozolomide-resistant malignant glioma. J Clin Oncol 27: 1262-1267, 2009. 
17. Bobola MS, Tseng SH, Blank A, et al: Role of O6-methylguanine-DNA methyltransferase in resistance of human brain tumor cell lines to the clinically relevant methylating agents temozolomide and streptozotocin. Clin Cancer Res 2: 735-741, 1996.

18. Turriziani M,Caporaso P,Bonmassar L, etal: O6-(4-bromothenyl) guanine (PaTrin-2), a novel inhibitor of O6-alkylguanine DNA alkyl-transferase, increases the inhibitory activity of temozolomide against human acute leukaemia cells in vitro. Pharmacol Res 53: 317-23, 2006.

19. Zhang J, Stevens MF, Laughton CA, et al: Acquired resistance to temozolomide in glioma cell lines: molecular mechanisms and potential translational applications. Oncology 78: 103-114, 2010 .

20. Weaver KD, Yeyeodu S, Cusack JC, et al: Potentiation of chemotherapeutic agents following antagonism of nuclear factor kappa B in human gliomas. J Neurooncol 61: 187-196, 2003.
21. Brandes AA, Franceschi E, Tosoni A, et al: O(6)-methylguanine DNA-methyltransferase methylation status can change between first surgery for newly diagnosed glioblastoma and second surgery for recurrence: clinical implications. Neuro Oncol 12: 283-288, 2010

22. Jung TY, Jung S, Moon KS, et al: Changes of the O6-methylguanine-DNA methyltransferase promoter methylation and MGMT protein expression after adjuvant treatment in glioblastoma. Oncol Rep 23: 1269-1276, 2010.

23. Blough MD, Westgate MR, Beauchamp D, et al: Sensitivity to temozolomide in brain tumor initiating cells. Neuro Oncol 12: 756-760, 2010.

24. Pistollato F, Abbadi S, Rampazzo E, et al: Intratumoral hypoxic gradient drives stem cells distribution and MGMT expression in glioblastoma. Stem Cells 28: 851-862, 2010. 